\title{
Representation of higher even-dimensional rhotrix
}

\author{
A. O. Isere \\ Department of Mathematics, Ambrose Alli University \\ Ekpoma, 310001, Nigeria \\ e-mails: abednis@yahoo.co.uk, \\ isereao@aauekpoma.edu.ng
}

Received: 13 July 2018

Revised: 25 December 2018

Accepted: 1 February 2019

\begin{abstract}
The multiplication of higher even-dimensional rhotrices is presented and generalized. The concept of empty rhotrix, and the necessary and sufficient conditions for an even-dimensional rhotrix to be represented over a linear map, are investigated and presented.
\end{abstract}

Keywords: Even-dimensional rhotrix, Representation, Empty rhotrix, Multiplication, Linear map.

2010 Mathematics Subject Classification: 15B99.

\section{Introduction}

A rhotrix is an arrangement of numbers in a rhomboid shape. This is similar to a matrix, which is an arrangement of numbers in a rectangular form. Rhotrix was first introduced by Ajibade [1], as an extension of the idea suggested by Atanassov and Shannon [4] in their work titled "matrix -tertions and matrix-noitrets". A formal definition of a real rhotrix as presented in the maiden paper is given below:

Definition 1.1. [1] A real rhotrix set of dimension three, denoted as $\hat{R}_{3}(\mathfrak{R})$ is defined as:

$$
\hat{R}_{3}(\mathfrak{R})=\left\{\left\langle\begin{array}{lll}
a \\
b & c & d \\
& e
\end{array}\right\rangle: a, b, c, d, e \in \mathfrak{R}\right\}
$$

where $c=h(R)$ is called the heart of any rhotrix $R$ belonging to $\hat{R}_{3}(\mathfrak{R})$ (a set of all real rhotrices of dimension 3) and $\mathfrak{R}$ is the set of real numbers. 
Examples showing extension of this set and analysis are copious in literature. A few are presented in these references $[2,3,5-7,10-14,18,19]$. It has been noted that these heart-oriented rhotrices are always of odd dimension. Thus, a rhotrix with even dimension is recently being introduced by Isere $[8,9]$. The algebra and analysis establishing this new structure as mathematically tractable were all presented in [9]. The heart-based rhotrices are classified as classical rhotrices, while even-dimensional rhotrices are classified as non-classical rhotrices [8].

Mean while, the addition and multiplication of heart-based rhotrices ( $h$-rhotrices) were first presented in [1]. Thus, addition and multiplication of two heart-based rhotrices are defined as:

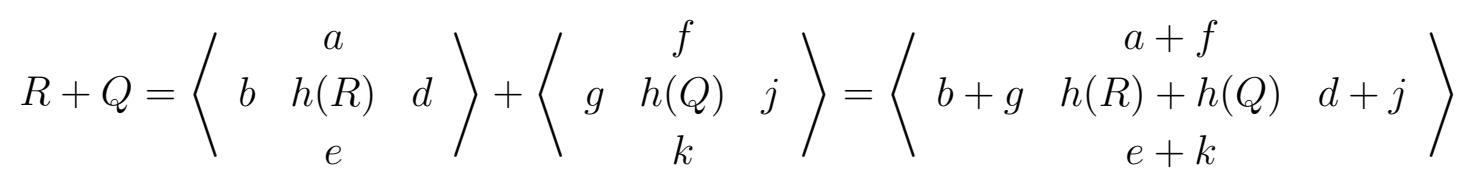

and

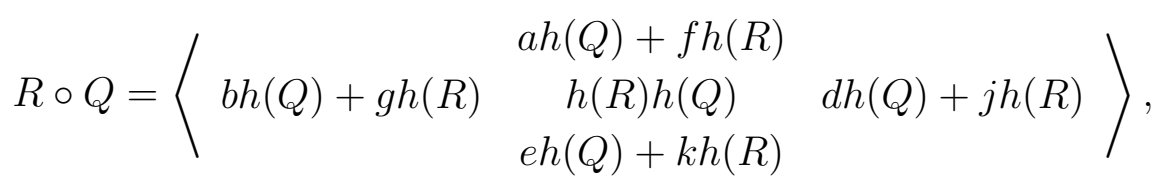

respectively. A generalization of this hearty multiplication is given in [14] and in [6]. A rowcolumn multiplication of heart-based rhotrices was proposed by Sani [15] as:

$$
R \circ Q=\left\langle\begin{array}{cc}
a f+d g \\
b f+e g & h(R) h(Q) \\
b j+e k
\end{array} \quad a j+d k\right\rangle .
$$

A generalization of this row-column multiplication was also later given by Sani [16] as:

$$
R_{n} \circ Q_{n}=\left\langle a_{i j}, c_{i j}\right\rangle \circ\left\langle b_{i j}, d_{l k}\right\rangle=\left\langle\sum_{i, j=1}^{t}\left(a_{i j} b_{i j}\right), \sum_{l, k=1}^{t-1}\left(c_{l k} d_{l k}\right)\right\rangle, t=(n+1) / 2,
$$

where $R_{n}$ and $Q_{n}$ are $n$-dimensional rhotrices (with $n$ rows and $n$ columns). These two methods of multiplication of rhotrices are very popular in literature. In both methods, the heart plays a significant role as shown above. A lot of work has been done on $h$-rhotrices. These works are also well known in literature, such as the conversion of a rhotrix into a coupled matrix by Sani [17]. A generalization of rhotrix was introduced as paraletrix by Aminu and Michael [3]. This concept shows more flexibility in mathematical arrays of numbers, where the number of rows and columns need not be the same. It was noted that not every paraletrix has a heart. Consequently, a rhotrix without a heart was introduced in $[8,9]$ as heartless rhotrices (hl-rhotrices). Such rhotrices were found to be even-dimensional. The simplest non-trivial even-dimensional rhotrix is of dimension two, and it is stated below:

Definition 1.2. A real rhotrix of dimension two is given as

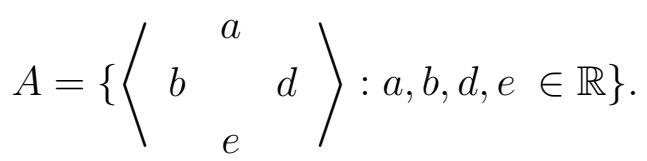


It is to be noted that an $n$-dimensional rhotrix with $n$ being even has its cardinality as $\left|R_{n}\right|=$ $\frac{1}{2}\left(n^{2}+2 n\right) \quad \forall n \in 2 N$. The multiplication of $h$-rhotrices, as remarked in [1], can be done in many ways. This is also true with even-dimensional rhotrices. In this work, we define multiplication of two even-dimensional rhotrices elementwise as follows:

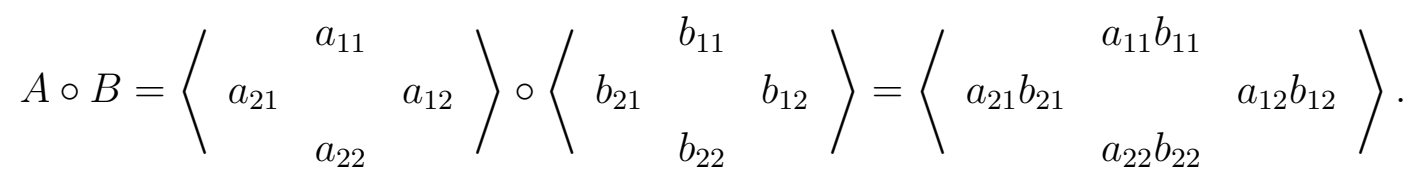

Moreover, we shall be looking at multiplication of higher even-dimensional rhotrices, the concept of empty rhotrix and the representation of an even-dimensional rhotrix over a linear map. The concept of rhotrix linear transformation was first investigated by Mohammed et al [13]. The necessary and sufficient conditions for a rhotrix to be represented by a linear map were given in [13]. It is to be noted that the rhotrix investigated was an $h$-rhotrix. These conditions will be stated in the next section. However, an extension of these conditions will be considered in this work, and the necessary and sufficient conditions for an even-dimensional rhotrix to be represented by a linear map will be presented.

\section{Preliminaries}

Some definitions will be considered in this section that will be useful in achieving the results anticipated in this work.

Definition 2.1. [13] A rhotrix $R$ of dimension $n$ is given as:

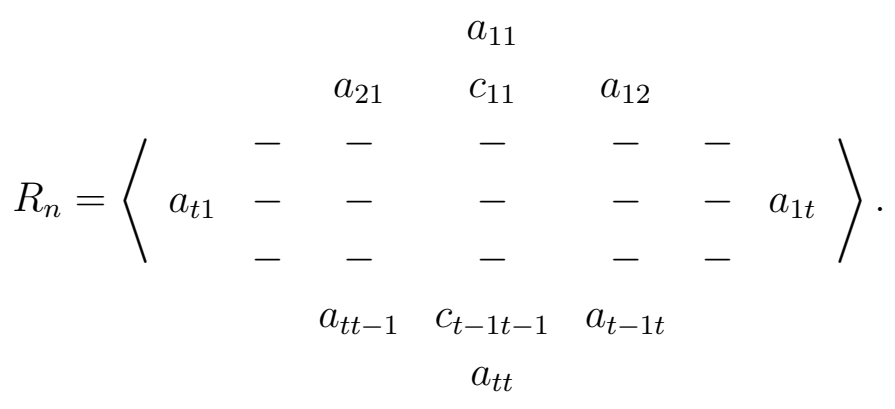

The element $a_{i j}(i, j=1,2, \ldots, t)$ and $c_{k l}(k, l=1,2, \ldots t-1)$ are called the major and minor entries of $R$, respectively. This is usually denoted as $R_{n}=\left\langle a_{i j}, c_{k l}\right\rangle$.

Definition 2.2. [13] Let $R_{n}=\left\langle a_{i j}, c_{k l}\right\rangle$ be an $n$ dimensional rhotrix. Then, $a_{i j}$ is the $(i, j)$ entries called the major entries of $R_{n}$ and $c_{k l}$ is the $(k, l)$-entries called the minor entries of $R_{n}$.

Definition 2.3. [16] A rhotrix $R_{n}=\left\langle a_{i j}, c_{k l}\right\rangle$ of $n$ dimension is a couple of two matrices $\left(a_{i j}\right)$ and $\left(c_{k l}\right)$ consisting of its major and minor matrices of $R_{n}$.

Definition 2.4. [13] Let $R_{n}=\left\langle a_{i j}, c_{k l}\right\rangle$ be an $n$ dimensional rhotrix. Then, rows and columns of $a_{i j}\left(c_{k l}\right)$ will be called the major (minor) rows and columns of $R_{n}$, respectively. 
Definition 2.5. [13] For any odd integer $n$, a $n \times n$ matrix $\left(a_{i j}\right)$ is called a filled coupled matrix if $a_{i j}=0$ for all $i, j$ whose sum $i+j$ is odd. We shall refer to these entries as the null entries of the filled coupled matrix.

Remark 2.1. ( $i) R_{n}=\left\langle a_{i j}, c_{k l}\right\rangle$ is a representation of any rhotrix. (ii) Moreover, an evendimensional rhotrix can also be represented as $R_{n}=\left\langle a_{i j}, c_{k l}\right\rangle$ or simply as $R_{n}=\left\langle a_{i},\right\rangle$. (iii) a $(n \times n)$ filled coupled matrix has $n^{2}$ entries.

Definition 2.6. For any odd integer $n$, $a(n \times n)$ matrix $\left(a_{i j}\right)$ is called a completely filled coupled matrix if $a_{i j}=0$ for all $i, j$ whose sum $i+j$ is odd and for all $i=j=\frac{n+1}{2}$. The entry corresponding to $a_{i j}=0, i=j=\frac{n+1}{2}$ is a special null-entry called the null entry of the completely filled coupled matrix.

Definition 2.7. The entries $a_{i j}$ whose sum $i+j$ is even, except when $i=j=\frac{n+1}{2} \quad \forall n \in 2 Z^{+}+1$, are called the real entries of the completely filled coupled matrix.

Theorem 2.1. [13] Let $n \in 2 Z^{+}+1$ and $F$ be a field. Then, a linear map $T: F^{n} \mapsto F^{n}$ can be represented by a rhotrix with respect to the standard basis if and only if $T$ is defined as:

$$
\begin{aligned}
T\left(x_{1}, y_{1}, x_{2}, y_{2}, \ldots, y_{t-1}, x_{t}\right)= & \left(\alpha_{1}\left(x_{1}, x_{2}, \ldots, x_{t}\right), \beta_{1}\left(y_{1}, y_{2}, \ldots, y_{t-1}\right),\right. \\
& \alpha_{2}\left(x_{1}, x_{2}, \ldots, x_{t}\right), \beta_{2}\left(y_{1}, y_{2}, \ldots, y_{t-1}\right), \ldots \\
& \left.\beta_{t-1}\left(y_{1}, y_{2}, \ldots, y_{t-1}\right), \alpha_{t}\left(x_{1}, x_{2}, \ldots, x_{t}\right)\right)
\end{aligned}
$$

where $t=\frac{n+1}{2}, \quad \alpha_{1}, \alpha_{2}, \ldots, \alpha_{t}$ and $\beta_{1}, \beta_{2}, \ldots, \beta_{t-1}$ are any linear maps on $F^{t}$ and $F^{t-1}$, respectively.

Lemma 2.2. Let $\left[a_{i j}\right]_{n}$ be $a(n \times n)$ filled coupled matrix, then:

(a) The number of all the real entries is given as

$$
\Pi_{n}=\frac{1}{2}\left(n^{2}+1\right) \quad \forall n \in 2 Z^{+}+1
$$

(b) The number of all the null entries is given as

$$
\emptyset_{n}=\frac{1}{2}\left(n^{2}-1\right) \quad \forall n \in 2 Z^{+}+1
$$

Proof. Since a $(n \times n)$ filled coupled matrix has $n^{2}$ entries, then $(a)+(b)=n^{2}$. Consider:

$$
\frac{1}{2}\left(n^{2}+1\right)+\frac{1}{2}\left(n^{2}-1\right)=n^{2}
$$

Then, $(a)$ and $(b)$ are true.

Remark 2.2. $\Pi_{n}+\emptyset_{n}$ as in Lemma 2.2 is an odd-dimensional rhotrix, i.e., the real entries are odd.

Lemma 2.3. Let $\left[a_{i j}\right]_{n}$ be a completely filled coupled matrix, then: 
(a) The number of all the real entries is given as

$$
\Pi_{n}=\frac{1}{2}\left(n^{2}-1\right) \quad \forall n \in 2 Z^{+}+1
$$

(b) The number of all the null entries is given as

$$
\emptyset_{n}=\frac{1}{2}\left(n^{2}+1\right) \quad \forall n \in 2 Z^{+}+1
$$

Proof. The proof is similar to the proof of Lemma 2.2 above.

Remark 2.3. $\Pi_{n}+\emptyset_{n}$ as in Lemma 2.3 is an even-dimensional rhotrix, i.e., the real entries are even.

Theorem 2.4. There is a one-to-one correspondence between the set of all n-dimensional rhotrices over a field $F$ and the set of all $n \times n$ completely filled coupled matrices over $F$.

Proof. The proof follows from Lemma 2.3 and the fact that any $n$-dimensional rhotrix is $n^{2}$ entries.

Remark 2.4. ( $(i)$ The set of all real entries $\left(\Pi_{n}\right)$ of the completely filled coupled matrix corresponds to the entries of an even-dimensional rhotrix $R_{n}=\left\langle a_{i j}, c_{k l}\right\rangle$ or simply as $R_{n}=\left\langle a_{i}\right\rangle$.

(ii) A filled coupled matrix and a completely filled coupled matrix comprise of both real and null entries.

(iii) All heart-based rhotrices result in a filled coupled matrix while all even-dimensional rhotrices result in a completely filled coupled matrix.

\section{Main Results}

This section presents the main results starting with the concept of empty rhotrix, then some examples of filled and completely filled coupled matrices and multiplication of higher evendimensional rhotrices.

\subsection{The concept of empty rhotrix}

Definition 3.1. A rhotrix that has no entry is an empty rhotrix, e.g., $A=\langle\rangle$.

Lemma 3.1. An empty rhotrix $A$ of $n$-th dimension contains null-entry of a completely- filled matrix as its only entry.

Proof. Recall that for an even-dimensional rhotrix $\left|R_{n}\right|=\frac{1}{2}\left(n^{2}+2 n\right) \quad \forall n \in 2 \mathbb{N}$. Since $n \in 2 \mathbb{N}$ implies that $0 \in 2 \mathbb{N}$ and $R_{0}=\langle\rangle$. The proof follows.

Corollary 3.1.1. An empty real rhotrix is even-dimensional. 
Proof. We prove by contradiction. Let $R_{n}$ be any $n$-dimensional real rhotrix. Suppose, $n$ is odd, then, its cardinality can be represented as

$$
\left|R_{n}\right|=\frac{1}{2}\left(n^{2}+1\right) n \in 2 \mathbb{Z}^{+}+1 .
$$

Since, an empty rhotrix has no entry, its cardinality is zero. That is

$$
0=\frac{1}{2}\left(n^{2}+1\right)
$$

implies that $n= \pm i$. Then, we have a contradiction. Now, suppose that $n$ is even, then

$$
\left|R_{n}\right|=\frac{1}{2}\left(n^{2}+2 n\right) n \in 2 \mathbb{N}
$$

implies that $n=0 \in 2 \mathbb{N}$. Thus, an empty rhotrix is even-dimensional.

Remark 3.1. $\mathbb{N}$ is a set of non-negative integers

\subsection{Some examples of filled and completely filled coupled matrices}

Example 3.1. A rhotrix of dimension five $\left(R_{5}\right)$ is given by:

$$
R_{5}=\left\langle\begin{array}{lllll} 
& a_{11} & & \\
& a_{21} & c_{11} & a_{12} & \\
a_{31} & c_{21} & a_{22} & c_{12} & a_{13} \\
& a_{32} & c_{22} & a_{23} &
\end{array}\right\rangle
$$

Then its corresponding filled coupled matrix is presented below:

$$
M\left(R_{5}\right)=\left[\begin{array}{ccccc}
a_{11} & 0 & a_{12} & 0 & a_{13} \\
0 & c_{11} & 0 & c_{12} & 0 \\
a_{21} & 0 & a_{22} & 0 & a_{23} \\
0 & c_{21} & 0 & c_{22} & 0 \\
a_{31} & 0 & a_{32} & 0 & a_{33}
\end{array}\right]
$$

Example 3.2. A rhotrix of dimension seven $\left(R_{7}\right)$ is given by:

$$
R_{7}=\left\langle\begin{array}{llllllll}
\multicolumn{8}{c}{a_{11}} \\
& a_{21} & c_{11} & a_{12} & & \\
a_{31} & c_{21} & a_{22} & c_{12} & a_{13} & \\
a_{41} & c_{31} & a_{32} & c_{22} & a_{23} & c_{13} & a_{14}
\end{array}\right.
$$

Then its corresponding filled coupled matrix will be presented below: 


$$
M\left(R_{7}\right)=\left[\begin{array}{ccccccc}
a_{11} & 0 & a_{12} & 0 & a_{13} & 0 & a_{14} \\
0 & c_{11} & 0 & c_{12} & 0 & c_{13} & 0 \\
a_{21} & 0 & a_{22} & 0 & a_{23} & 0 & a_{24} \\
0 & c_{21} & 0 & c_{22} & 0 & c_{23} & 0 \\
a_{31} & 0 & a_{32} & 0 & a_{33} & 0 & a_{34} \\
0 & c_{31} & 0 & c_{32} & 0 & c_{33} & 0 \\
a_{41} & 0 & a_{42} & 0 & a_{43} & 0 & a_{44}
\end{array}\right]
$$

Example 3.3. A rhotrix of dimension four $\left(R_{4}\right)$ is given by:

$$
R_{4}=\left\langle\begin{array}{lllll} 
& a_{11} & & \\
& a_{21} & c_{11} & a_{12} & \\
a_{31} & c_{21} & & c_{12} & a_{13}
\end{array}\right\rangle
$$

Then its corresponding completely filled coupled matrix is presented below:

$$
C\left(R_{4}\right)=\left[\begin{array}{ccccc}
a_{11} & 0 & a_{12} & 0 & a_{13} \\
0 & c_{11} & 0 & c_{12} & 0 \\
a_{21} & 0 & 0^{*} & 0 & a_{23} \\
0 & c_{21} & 0 & c_{22} & 0 \\
a_{31} & 0 & a_{32} & 0 & a_{33}
\end{array}\right]
$$

Example 3.4. A rhotrix of dimension six $\left(R_{6}\right)$ is given by:

$$
R_{6}=\left\langle\begin{array}{llllllll}
\multicolumn{8}{c}{a_{11}} \\
& a_{21} & c_{11} & a_{12} & & \\
a_{31} & c_{21} & a_{22} & c_{12} & a_{13} & \\
a_{41} & c_{31} & a_{32} & & a_{23} & c_{13} & a_{14}
\end{array}\right.
$$

Then its corresponding completely filled coupled matrix is:

$$
C\left(R_{6}\right)=\left[\begin{array}{ccccccc}
a_{11} & 0 & a_{12} & 0 & a_{13} & 0 & a_{14} \\
0 & c_{11} & 0 & c_{12} & 0 & c_{13} & 0 \\
a_{21} & 0 & a_{22} & 0 & a_{23} & 0 & a_{24} \\
0 & c_{21} & 0 & 0^{*} & 0 & c_{23} & 0 \\
a_{31} & 0 & a_{32} & 0 & a_{33} & 0 & a_{34} \\
0 & c_{31} & 0 & c_{32} & 0 & c_{33} & 0 \\
a_{41} & 0 & a_{42} & 0 & a_{43} & 0 & a_{44}
\end{array}\right]
$$

Remark 3.2. A completely filled coupled matrix is obtained from even-dimensional rhotrix, and contains the null-entry of the completely filled coupled matrix denoted as $0^{*}$, while a filled coupled matrix is obtained from odd-dimensional rhotrices. 


\subsection{Multiplication of higher even-dimensional rhotrices}

Multiplication of higher even-dimensional rhotrices whether even or odd dimensional can be defined in many ways. In this work, elementwise multiplication method is presented for higher even-dimensional rhotrices. Examples of rhotrices of dimension four are presented for the purpose of demonstration. Let

$$
A=\left\langle\begin{array}{lllll} 
& a_{2} & a_{3} & a_{4} & \\
a_{5} & a_{6} & & a_{7} & a_{8}
\end{array}\right\rangle, \quad B=\left\langle\begin{array}{cccccc} 
& b_{2} & b_{3} & b_{4} & \\
b_{5} & b_{6} & & b_{7} & b_{8} \\
& b_{9} & b_{10} & b_{11} &
\end{array}\right\rangle
$$

then

$$
\begin{aligned}
& a_{1} b_{1} \\
& A \odot B=\left\langle\begin{array}{lcccc} 
& a_{2} b_{2} & a_{3} b_{3} & a_{4} b_{4} & \\
a_{5} b_{5} & a_{6} b_{6} & & a_{7} b_{7} & a_{8} b_{8} \\
& a_{9} b_{9} & a_{10} b_{10} & a_{11} b_{11} &
\end{array}\right\rangle \\
& a_{12} b_{12}
\end{aligned}
$$

Example 3.5. Let

$$
A=\left\langle\begin{array}{lllll}
3 & 1 & 4 & \\
5 & 6 & & 7 & 8 \\
9 & 10 & 5
\end{array}\right\rangle, \quad B=\left\langle\begin{array}{lllll}
2 & 4 & 1 & \\
7 & 8 & & 9 & 5 \\
6 & 8 & 3 &
\end{array}\right\rangle
$$

then

$$
A \odot B=\left\langle\begin{array}{rrrrr}
3 \odot 2 & 1 \odot 4 & 4 \odot 1 \\
5 \odot 7 & 6 \odot 8 & & 7 \odot 9 & 8 \odot 5 \\
9 \odot 6 & 10 \odot 8 & 5 \odot 3 \\
& 3 \odot 10
\end{array}\right.
$$

Generally, a rhotrix $R$ of dimension $n$ ( $n$ being even) can be written as:

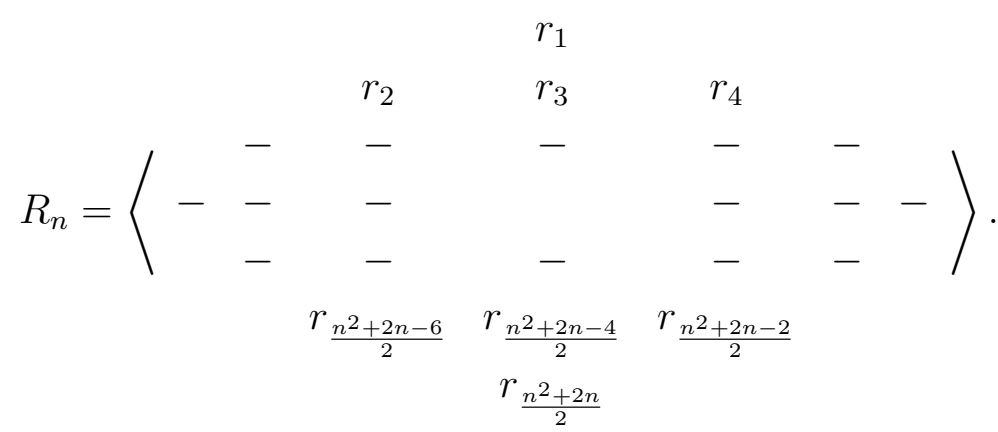


A generalization of the elementwise multiplication of even-dimensional rhotrices is as follows. Let $R_{n}=\left\langle a_{i}\right\rangle$ and $Q_{n}=\left\langle b_{j}\right\rangle$, be two even-dimensional rhotrices, then their multiplication is as follows

$R_{n} \odot Q_{n}=\left\langle a_{i}\right\rangle \odot\left\langle b_{j}\right\rangle=\left\langle\sum_{i=1}^{t} a_{i}\right\rangle \odot\left\langle\sum_{j=1}^{t} b_{j}\right\rangle=\left\langle\sum_{k=1}^{t}\left(a_{k} b_{k}\right)\right\rangle, t=\left(n^{2}+2 n\right) / 2, n \in 2 \mathbb{N}$,

where the product $\left(a_{i j} b_{i j}\right)$ is empty whenever $i=j=\frac{t+1}{2} \forall t \in 2 Z^{+}+1$.

\section{Linear maps on an even-dimensional rhotrix}

The concept of representation by a linear map helps to establish the existence of a linear structure. In this section, we investigate the representation of an even-dimensional rhotrix over a linear map.

Theorem 4.1. Let $n \in 2 Z^{+}+1$ and $F$ be a field. Then, a linear map $\tau: F^{n} \mapsto F^{n}$ can be represented by an even-dimensional rhotrix with respect to the standard basis if and only if $\tau$ is defined as:

$$
\begin{aligned}
\tau\left(x_{1}, y_{1}, x_{2}, y_{2}, \ldots, y_{t-1}, x_{t}\right)= & \left(\alpha_{1}\left(x_{1}, x_{2}, \ldots, x_{t}\right), \beta_{1}\left(y_{1}, y_{2}, \ldots, y_{t-1}\right),\right. \\
& \alpha_{2}\left(x_{1}, x_{2}, \ldots, x_{t}\right), \beta_{2}\left(y_{1}, y_{2}, \ldots, y_{t-1}\right), \ldots, \\
& \beta_{\frac{t}{2}}\left(y_{1}, y_{2}, \ldots, 0\left(y_{\frac{t}{2}}\right), \ldots, y_{t-1}\right) \forall t-1 \in 2 Z^{+}+1, \\
& \alpha_{\frac{t+1}{2}}\left(x_{1}, x_{2}, \ldots, 0\left(x_{\frac{t+1}{2}}\right), \ldots, x_{t}\right) \forall t \in 2 Z^{+}+1, \ldots, \\
& \left.\beta_{t-1}\left(y_{1}, y_{2}, \ldots, y_{t-1}\right), \alpha_{t}\left(x_{1}, x_{2}, \ldots, x_{t}\right)\right),
\end{aligned}
$$

where $t=\frac{n+2}{2}, \alpha_{1}, \alpha_{2}, \ldots, \alpha_{\frac{t+1}{2}}, \ldots, \alpha_{t}$ and $\beta_{1}, \beta_{2}, \ldots, \beta_{\frac{t}{2}}, \ldots, \beta_{t-1}$ are any linear maps on $F^{t}$ and $F^{t-1}$, respectively.

Proof. Case 1 (when $t \in 2 Z^{+}+1$ ).

Given that

$$
\begin{aligned}
\tau\left(x_{1}, y_{1}, x_{2}, y_{2}, \ldots, y_{t-1}, x_{t}\right)= & \left(\alpha_{1}\left(x_{1}, x_{2}, \ldots, x_{t}\right), \beta_{1}\left(y_{1}, y_{2}, \ldots, y_{t-1}\right),\right. \\
& \alpha_{2}\left(x_{1}, x_{2}, \ldots, x_{t}\right), \beta_{2}\left(y_{1}, y_{2}, \ldots, y_{t-1}\right), \ldots, \\
& \beta_{\frac{t}{2}}\left(y_{1}, y_{2}, \ldots, 0\left(y_{\frac{t}{2}}\right), \ldots, y_{t-1}\right) \forall t-1 \in 2 Z^{+}+1, \\
& \alpha_{\frac{t+1}{2}}\left(x_{1}, x_{2}, \ldots, 0\left(x_{\frac{t+1}{2}}\right), \ldots, x_{t}\right) \forall t \in 2 Z^{+}+1, \ldots, \\
& \left.\beta_{t-1}\left(y_{1}, y_{2}, \ldots, y_{t-1}\right), \alpha_{t}\left(x_{1}, x_{2}, \ldots, x_{t}\right)\right)
\end{aligned}
$$

where $t=\frac{n+2}{2}, \alpha_{1}, \alpha_{2}, \ldots, \alpha_{\frac{t+1}{2}}, \ldots, \alpha_{t}$ and $\beta_{1}, \beta_{2}, \ldots, \beta_{\frac{t}{2}}, \ldots, \beta_{t-1}$ are any linear maps on $F^{t}$ and $F^{t-1}$, respectively. 
Now let us consider the standard basis:

$$
\begin{aligned}
\tau\left(e_{1}\right)= & {\left[\alpha_{1}(1,0, \ldots, 0), 0, \ldots, \alpha_{t}(1,0 \ldots, 0)\right] } \\
\tau\left(e_{1}\right)= & {\left[0, \beta_{1}(1,0, \ldots, 0), 0, \ldots, \beta_{t-1}(1,0 \ldots, 0)\right] } \\
& \vdots \\
\tau\left(e_{1}\right)= & {\left[\alpha_{1}(1,0, \ldots, 0), 0, \ldots, \alpha_{\frac{t+1}{2}}\left(0 \ldots, 0\left(x_{\frac{t+1}{2}}\right), \ldots 0\right), \ldots, \alpha_{t}(1,0 \ldots, 0)\right] } \\
& \vdots \\
\tau\left(e_{t}\right) & =\left[0, \beta_{1}(0, \ldots, t), 0, \ldots, \beta_{t-1}(0 \ldots, 0), 1\right] \\
\tau\left(e_{t}\right) & =\left[\alpha_{1}(0, \ldots, t), 0, \ldots, \alpha_{t}(0 \ldots, 1)\right]
\end{aligned}
$$

Putting the above linear equations into a matrix, we have

$$
\left[\begin{array}{ccccccc}
\alpha_{11} & 0 & \alpha_{12} & \ldots & \alpha_{1 t-1} & 0 & \alpha_{1 t} \\
0 & \beta_{11} & 0 & \ldots & 0 & \beta_{1 t-1} & o \\
\vdots & \vdots & \vdots & \ddots & \vdots & \vdots & \vdots \\
\alpha_{\frac{t+1}{2} 1} & 0 & \alpha_{\frac{t+1}{2} 2} & \ldots & 0 & \ldots & \alpha_{\frac{t+1}{2} t} \\
\vdots & \vdots & \vdots & \ddots & \vdots & \vdots & \vdots \\
0 & \beta_{t-11} & 0 & \ldots & 0 & \beta_{t-1 t-1} & 0 \\
\alpha_{t 1} & 0 & \alpha_{t 2} & \ldots & \alpha_{t t-1} & 0 & \alpha_{t t}
\end{array}\right]
$$

The transpose of the above matrix is the matrix of transformation denoted as

$$
m(\tau)=\left[\begin{array}{ccccccc}
\alpha_{11} & 0 & \alpha_{12} & \ldots & \alpha_{1 t-1} & 0 & \alpha_{1 t} \\
0 & \beta_{11} & 0 & \ldots & 0 & \beta_{1 t-1} & 0 \\
\vdots & \vdots & \vdots & \ddots & \vdots & \vdots & \vdots \\
\alpha_{\frac{t+1}{2} 1} & 0 & \alpha_{\frac{t+1}{2} 2} & \ldots & 0 & \ldots & \alpha_{\frac{t+1}{2} t} \\
\vdots & \vdots & \vdots & \ddots & \vdots & \vdots & \vdots \\
0 & \beta_{t-11} & 0 & \ldots & 0 & \beta_{t-1 t-1} & 0 \\
\alpha_{t 1} & 0 & \alpha_{t 2} & \ldots & \alpha_{t t-1} & 0 & \alpha_{t t}
\end{array}\right]^{T}
$$

The result is a completely filled coupled matrix from which we have the even-dimensional rhotrix representation.

Conversely, suppose that $\tau: F^{n} \mapsto F^{n}$ has an even-dimensional rhotrix representation $\left\langle\alpha_{i j}, \beta_{k l}\right\rangle$ in the standard basis. Then, the corresponding matrix representation of $\tau$ is the com- 
pletely filled coupled matrix given above. From this, we obtain the linear system below:

$$
\begin{aligned}
\tau\left(e_{1}\right) & =\left[\alpha_{1}(1,0, \ldots, 0), 0, \ldots, \alpha_{t}(1,0 \ldots, 0)\right] \\
\tau\left(e_{1}\right) & =\left[0, \beta_{1}(1,0, \ldots, 0), 0, \ldots, \beta_{t-1}(1,0 \ldots, 0)\right] \\
& \vdots \\
\tau\left(e_{1}\right) & =\left[\alpha_{1}(1,0, \ldots, 0), 0, \ldots, \alpha_{\frac{t+1}{2}}\left(0 \ldots, 0\left(x_{\frac{t+1}{2}}\right), \ldots 0\right), \ldots, \alpha_{t}(1,0 \ldots, 0)\right] \\
& \vdots \\
\tau\left(e_{t}\right) & =\left[0, \beta_{1}(0, \ldots, t), 0, \ldots, \beta_{t-1}(0 \ldots, 0), 1\right] \\
\tau\left(e_{t}\right) & =\left[\alpha_{1}(0, \ldots, t), 0, \ldots, \alpha_{t}(0 \ldots, 1)\right]
\end{aligned}
$$

Case 1 (when $t-1 \in 2 Z^{+}+1$ ).

The proof follows similarly.

Remark 4.1. The above theorem is seeing our even-dimensional rhotrix as a completely filled couple matrix.

Example 4.1. Consider the linear mapping $\tau: \mathfrak{R} \mapsto \mathfrak{R}$ defined by $\tau(x, y, z)=(a x+d z, 0, b x+$ ez). Find the hl-rhotrix represented by the linear transformation(linear map) $\tau$ with respect to the standard basis.

Solution:

$$
\begin{aligned}
\tau(1,0,0) & =(a, 0, b) \\
\tau(0,1,0) & =(0,0,0) \\
\tau(0,0,1) & =(d, 0, e)
\end{aligned}
$$

Then, putting this into matrix gives

$$
\left(\begin{array}{lll}
a & 0 & b \\
0 & 0 & 0 \\
d & 0 & e
\end{array}\right)
$$

Thus, the matrix of representation is the transpose of the above matrix

$$
m(\tau)=\left(\begin{array}{lll}
a & 0 & b \\
0 & 0 & 0 \\
d & 0 & e
\end{array}\right)^{T}=\left[\begin{array}{lll}
a & 0 & d \\
0 & 0 & 0 \\
b & 0 & e
\end{array}\right]
$$

which is a completely filled coupled matrix. Then the even-dimensional rhotrix by $\tau$ is

$$
R(\tau)=\left\langle\begin{array}{lll} 
& a & \\
b & & d
\end{array}\right\rangle
$$

Example 4.2. Consider the linear mapping $\tau: \mathfrak{R} \mapsto \mathfrak{R}$ defined by $\tau(a, b, c, d, e)=(a+2 c-$ $5 e, 3 b+6 d, 4 a+10 e, 8 b-11 d, 9 a+12 c+13 e)$. Find the even-dimensional rhotrix represented by the linear transformation(linear map) $\tau$ with respect to the standard basis. 
Solution:

$$
\begin{aligned}
& \tau(1,0,0,0,0)=(1,0,4,0,9) \\
& \tau(0,1,0,0,0)=(0,3,0,8,0) \\
& \tau(0,0,1,0,0)=(2,0,0,0,12) \\
& \tau(0,0,0,1,0)=(0,6,0,-11,0) \\
& \tau(0,0,0,0,1)=(-5,0,10,0,13)
\end{aligned}
$$

Thus, the matrix of representation is given below:

$$
m(\tau)=\left[\begin{array}{ccccc}
1 & 0 & 4 & 0 & 9 \\
0 & 3 & 0 & 8 & 0 \\
2 & 0 & 0 & 0 & 12 \\
0 & 6 & 0 & -11 & 0 \\
-5 & 0 & 10 & 0 & 13
\end{array}\right]^{T}=\left[\begin{array}{ccccc}
1 & 0 & 2 & 0 & -5 \\
0 & 3 & 0 & 6 & 0 \\
4 & 0 & 0 & 0 & 10 \\
0 & 8 & 0 & -11 & 0 \\
9 & 0 & 12 & 0 & 13
\end{array}\right]
$$

which is a completely filled coupled matrix. Then the even-dimensional rhotrix by $\tau$ is

$$
R(\tau)=\left\langle\begin{array}{ccccc} 
& 4 & 3 & 2 & \\
9 & 8 & & 6 & -5 \\
& 12 & -11 & 10 &
\end{array}\right\rangle
$$

13

This is an even-dimensional rhotrix of dimension 4.

Example 4.3. Consider the linear mapping $\tau: \mathfrak{R} \mapsto \mathfrak{R}$ defined by $\tau(a, b, c, d, e, f, g)=(3 a+$ $2 c-4 g-2 e, 5 b+4 d+3 f, 5 a-7 c+3 e-g, 8 b-5 f, 7 a+12 c-3 e+5 g,-4 b+2 d+f, a+14 c-7 e+10 g)$. Find the even-dimensional rhotrix represented by the linear transformation(linear map) $\tau$ with respect to the standard basis.

Solution:

$$
\begin{aligned}
& \tau(1,0,0,0,0,0,0)=(3,0,5,0,7,0,1) \\
& \tau(0,1,0,0,0,0,0)=(0,5,0,8,0,-4,0) \\
& \tau(0,0,1,0,0,0,0)=(2,0,-7,0,12,0,14) \\
& \tau(0,0,0,1,0,0,0)=(0,4,0,0,0,2,0) \\
& \tau(0,0,0,0,1,0,0)=(-2,0,3,0,-3,0,-7) \\
& \tau(0,0,0,0,1,0,0)=(0,3,0,-5,0,1,0) \\
& \tau(0,0,0,0,1,0,0)=(-4,0,-1,0,5,0,10)
\end{aligned}
$$

Thus, the matrix of representation is given below:

$$
m(\tau)=\left[\begin{array}{ccccccc}
3 & 0 & 5 & 0 & 7 & 0 & 1 \\
0 & 5 & 0 & 8 & 0 & -4 & 0 \\
2 & 0 & -7 & 0 & 12 & 0 & 14 \\
0 & 4 & 0 & 0 & 0 & 2 & 0 \\
-2 & 0 & 3 & 0 & -3 & 0 & -7 \\
0 & 3 & 0 & -5 & 0 & 1 & 0 \\
-4 & 0 & -1 & 0 & 5 & 0 & 10
\end{array}\right]^{T}=\left[\begin{array}{ccccccc}
3 & 0 & 2 & 0 & -2 & 0 & -4 \\
0 & 5 & 0 & 4 & 0 & 3 & 0 \\
5 & 0 & -7 & 0 & 3 & 0 & -1 \\
0 & 8 & 0 & 0 & 0 & -5 & 0 \\
7 & 0 & 12 & 0 & -3 & 0 & 5 \\
0 & -4 & 0 & 2 & 0 & 1 & 0 \\
1 & 0 & 14 & 0 & -7 & 0 & 10
\end{array}\right]
$$


which is a completely filled coupled matrix. Then the even-dimensional rhotrix by $\tau$ is

$$
R(\tau)=\left\langle\begin{array}{cccccccc} 
& & & 3 & & & \\
& & 5 & 5 & 2 & & \\
1 & 8 & -7 & 4 & -2 & \\
& -4 & 12 & & 3 & 3 & -4
\end{array}\right\rangle
$$

This is an even-dimensional rhotrix of dimension 6 .

\section{Conclusion}

A strenuous effort was made to represent an even-dimensional rhotrix over a linear map. This representation showed that an even-dimensional rhotrix is a linear structure, and that it is a special type of rhotrix. All even-dimensional rhotrices are rhotrices except for the converse. Representing a rhotrix this way enables us to have by definition, even-dimensional rhotrices. Therefore, this work is an expansion and a contribution to rhotrix algebra.

\section{Acknowledgements}

I want to acknowledge my late colleague Dr. E. E. David of the Department of Mathematics, University of Port-Harcout (who passed away when the work was almost concluded). His penetrating ideas and questions have moved me to investigate the representation of an even-dimensional rhotrix over a linear map as well as the concept of empty rhotrix. This came to the fore when I first presented "Even-dimensional rhotrix" during AAS/AMU International Symposium on New Trend in Mathematics, Abuja, July 2016.

\section{References}

[1] Ajibade, A. O. (2003). The concept of Rhotrix in Mathematical Enrichment, International Journal of Mathematical Education in Science and Technology, 34 (2), 175-177.

[2] Aminu, A. (2009). On the Linear System over Rhotrices, Notes on Number Theory and Discrete Mathematics, 15 (4), 7-12.

[3] Aminu, A. \& Michael, O. (2015). An introduction to the concept of paraletrix, a generalization of rhotrix, Journal of the African Mathematical Union \& Springer-Verlag, 26 (5-6), 871-885.

[4] Atanassov, K. T. \& Shannon, A. G. (1998). Matrix-Tertions and Matrix-Noitrets: Exercise for Mathematical Enrichment, International Journal Mathematical Education in Science and Technology, 29 (6), 898-903. 
[5] Chinedu, M. P. (2012). Row-Wise Representation of Arbitrary Rhotrix, Notes on Number Theory and Discrete Mathematics, 18 (2), 1-27.

[6] Ezugwu, E. A., Ajibade, A. O. \& Mohammed, A. (2011). Generalization of Heart-Oriented rhotrix Multiplication and its Algorithm Implementation, International Journal of Computer Applications, 13 (3), 5-11.

[7] Isere, A. O. (2016). Natural Rhotrix. Cogent Mathematics, 3 (1) , 1-10 (article 1246074).

[8] Isere, A. O. (2017). A note on the Classical and Non-Classical rhotrices, The Journal of Mathematical Association of Nigeria (Abacus), 44(2), 119-124.

[9] Isere, A. O. (2018). Even-dimensional rhotrix, Notes on Number Theory and Discrete Mathematics, 24 (2), 125-133.

[10] Isere, A. O. \& Adeniran, J. O. (2018). The Concept of Rhotrix Quasigroups and Rhotrix Loops, The Journal of Nigerian Mathematical Society, 37(3), 139-153.

[11] Mohammed, A. (2009). A remark on the classifications of rhotrices as abstract strutures, International Journal of Physical Sciences, 4 (9), 496-499.

[12] Mohammed, A. \& Tella, Y. (2012). Rhotrix Sets and Rhotrix Spaces Category, International Journal of Mathematics and computational methods in Science and Technology, 2 (5), 2012.

[13] Mohammed, A., Balarabe, M. \& Imam, A. T. (2012). Rhotrix Linear Transformation, Advances in Linear Algebra \& Matrix Theory, 2, 43-47.

[14] Mohammed, A. (2014) A new expression for rhotrix, Advances in Linear Algebra \& Matrix Theory, 4, 128-133.

[15] Sani, B. (2004). An alternative method for multiplication of rhotrices, International Journal of Mathematical Education in Science and Technology, 35, 777-781.

[16] Sani, B. (2007). The row-column multiplication for higher dimensional rhotrices, International Journal of Mathematical Education in Science and Technology, 38, 657-662.

[17] Sani, B. (2008). Conversion of a rhotrix to a coupled matrix, International Journal of Mathematical Education in Science and Technology, 39, 244-249.

[18] Tudunkaya, S. M. \& Manjuola, S. O. (2010). Rhotrices and the Construction of Finite Fields, Bull. Pure Appl Sci. Sect, E. Math. Stat., 29 (2), 225-229.

[19] Usaini, S. \& Mohammed, L. (2012). On the rhotrix eigenvalues and eigenvectors, Journal of the African Mathematical Union \& Springer-Verlag, 25, 223-235. 